\title{
A Educação Física na era Covid-19: as aulas remotas na graduação durante a pandemia
}

https://doi.org/10.11606/issn.1981-4690.v35i3p67-75

\section{Resumo}

Esta pesquisa teve por objetivo analisar o impacto das atividades remotas nos cursos de Educação Física daUniversidade da Região de Joinville/SC-Univille, duranteoperíodopandêmico.Ométodoadotado para investigação foi o descritivo e transversal. Participaram do estudo 110 estudantes, todos dos cursos de graduação em Educação Física da Univille. Como instrumento de pesquisa foi utilizado um questionário, organizado na plataforma Google Forms ${ }^{\circledR}$, composto de 12 questões afirmativas, no modelo Escala Likert paramensuraçãodas respostas. Asperguntasforamorganizadasemtrêscategorias:Aprendizado,Caminho ProfissionaleReconhecimentolntrínseco.Osdadosdepoisdecoletadosforamorganizadosemumaplanilha do programa SPSS ${ }^{\circledR}$ 16.0. Após a determinação da normalidade da distribuição dos dados, por meio do teste de Kolmogorov-Smirnov, foi aplicado o teste de correlação de Pearson e os valores de $p<0,05$ foram considerados significativos. Ao efetuarmos a análise estatística em cada categoria, os resultados revelaram cinco associações lineares significativas moderadas. Uma no domínio Aprendizado ( $p$-valor $<0,002$ ), duas no Caminho Profissional ( $p$-valor $<0,0003$ e $p$-valor $<0,003$ ), e duas no Reconhecimento Intrínseco ( $p$-valor $<0,005$ e $p$-valor $<0,002$ ). Para a análise entre categorias foram encontradas sete correlações moderadas. Duas no componente"AprendizadoeCaminho Profissional"( $p$-valor $<0,004$ e p-valor $<0,002$ ), três no"Aprendizado e Reconhecimento Intrínseco" ( $p$-valor $<0,005$, p-valor $<0,004$ e p-valor $<0,001$ ), e duas na"Caminho Profissional e Reconhecimento Intrínseco"com o mesmovalor ( $p$-valor $<0,003$ ). Diante das evidências encontradas, foi possível constatar por intermédio do predomínio de associações lineares significativas, queas atividades remotas dificultaramo processoensino-aprendizagem para osacadêmicos dos cursos de Educação Física, durante a pandemia.

Palavras-chave: Pandemia; Atividades Remotas; Educação Física; Graduação.

\section{Introdução}

A universidade é um espaço destinado à formação humanística e profissional, de expressão política e científica, especializada na transmissão e produção do conhecimento ${ }^{1}$.

À vista disso, o referido processo de transformação está fundamentado em três pilares que constituem a base de sustentação do ensino superior: ensino, pesquisa e extensão. $\mathrm{O}$ ensino é o meio pelo qual ocorre a concepção do saber, a pesquisa está relacionada a concretização deste saber, através da busca e resolução de um determinado problema, e a extensão associada ao ambiente acadêmico e sua inserção na comunidade, promovendo açóes educativas, culturais e científicas em prol da sociedade ${ }^{2}$.

Entretanto, em dezembro de 2019 foi relatada a existência do novo coronavírus (SARS-CoV-2), em Wuhan, na China, com repercussão mundial. Assim, em razão do seu alto índice de contágio, uma das medidas de combate tomadas a nível global foi o isolamento social ${ }^{3-4}$.

Devido a isso, várias instituiçôes de ensino
*Universidade Região de Joinville, Joinville, SC, Brasil. 
tiveram que se reinventar e se adequar a um novo método ${ }^{5}$, dando lugar ao ensino mediado por tecnologias, que incluíram plataformas de ensino e ou de web-conferências que poderiam ser acessadas por smartphones, tablets e notebooks. Este processo oportunizou o uso da internet em diferentes contextos e funcionalidades, tornando-se um novo desafio para a formação acadêmica ${ }^{6}$. Em consequência disso, as adaptaçóes impactaram de forma abrupta tanto estudantes, quanto professores, que juntos atribuíram uma experimentação atípica de ensinar e aprender a fim de manter a continuidade das açóes ${ }^{7}$.

Tendo em vista os fatos mencionados, a Universidade da Região de Joinville/SC - Univille, a partir do dia 16 de março de 2020, optou por permanecer acolhendo os acadêmicos para as atividades de ensino para o crescimento profissional, mesmo durante a pandemia, de forma remota, conforme possibilitou as normativas e regulamentação educacional nacional.

Desta forma, as aulas foram mediadas por tecnologias da informação e comunicação (TIC's), com o suporte de uma ferramenta denominada Microsoft Teams ${ }^{\odot}$. A referida plataforma auxiliou na comunicaçáo e combinou: bate-papo, videoconferências e compartilhamento de arquivos, para que assim, as aulas pudessem seguir normalmente no seu horário previsto. Esse formato oportunizou a transmissão de conteúdos e a realização de novas experiências de aprendizagem por meio de aulas síncronas, questionários, tarefas e discussóes, indispensáveis para o desenvolvimento do estudante ${ }^{8}$.

Contudo, mesmo a universidade fazendo seu papel de continuar com o ensino, temos como hipótese que a falta das aulas práticas pode prejudicar os acadêmicos dos cursos de Educação Física (Licenciatura e Bacharelado), uma vez que para esse curso a experimentação prática do exercício físico é tâo importante quanto o conhecimento teórico, auxiliando-os no desenvolvimento de atitudes profissionais? .

Com isso, esta pesquisa teve por objetivo analisar o impacto das atividades remotas nos cursos de Educação Física (Licenciatura e Bacharelado) da Universidade da Regiāo de Joinville/SC - Univille, durante o período pandêmico.

\section{Método}

Para esta pesquisa foi utilizado como modelo de investigaçáo o método observacional, descritivo e transversal, apoiados por uma revisão conceitual a partir de leituras de artigos científicos dentro da temática de estudo. Participaram do estudo 110 estudantes, todos cursando graduação em Educação Física (Licenciatura e Bacharelado) na Univille, localizada em Joinville, Santa Catarina - Brasil. A escolha dos candidatos foi intencional, ou seja, todos os acadêmicos que participaram das aulas remotas/virtualizadas foram convidados a participar do estudo.

$\mathrm{O}$ isolamento social, na regiāo de Joinville/SC iniciou no dia 16 de março, entretanto este estudo teve início em novembro, ou seja, final do ano letivo, devido às questóes relativas à liberação ética, e até mesmo para verificar o impacto desta medida para a formação acadêmica. Desta maneira, buscou-se reunir o maior número possível de estudantes, participantes nas diferentes disciplinas e séries ofertadas nos cursos de Educação Física durante o período que correspondeu ao processo de isolamento social.

Como instrumento de pesquisa foi utilizado um questionário especialmente desenvolvido para o estudo, pelos próprios pesquisadores. Este instrumento foi organizado na plataforma Google Forms ${ }^{\circledR}$ e foi composto de 12 questôes afirmativas, no modelo Escala Likert para mensuração das respostas. Logo, o participante poderia optar por: [1] concordo plenamente, [2] concordo, [3] não concordo nem discordo, [4] discordo e [5] discordo totalmente, de acordo com o que mais correspondesse ao seu grau de concordância (ou discordância) em relação à questão.

Para tabulação dos dados e posterior análise dos resultados, as afirmativas foram organizadas em três categorias: "Aprendizado", "Caminho Profissional" e "Reconhecimento Intrínseco", conforme evidenciado no QUADRO 1. A triagem destas categorias foram pautadas nas adversidades impostas pelo cenário pandêmico, cada domínio reflete os possíveis impactos com a mudança do ensino, em uma proposta que estabelece conexão entre os componentes, para assim, entender sob a ótica dos acadêmicos de Educação Física (Licenciatura e Bacharelado) essas alteraçóes.

Além disso, torna-se importante mencionar 
que para validar a referida ferramenta, foi aplicado um teste piloto no sentido de verificar e consolidar o processo de interação entre os interlocutores.

Como veículo de distribuição, foi utilizado meio virtual a partir dos aplicativos de redes sociais como WhatsApp e Instagram ${ }^{\oplus}$, bem como o e-mail institucional do curso. O Termo de Consentimento Livre e Esclarecido -TCLE antecede as questóes de pesquisa e para responderem ao formulário com as afirmativas seria necessário consentir a participação. Este instrumento tinha aproximadamente dez minutos para a sua conclusão, sendo de rápida leitura e compreensão. Foram utilizados os dados dos questionários preenchidos adequadamente e os demais foram excluídos.
As informações coletadas foram analisadas por meio da estatística descritiva (apresentadas em números absolutos, percentuais e moda), organizadas e disponibilizadas em uma planilha do programa Microsoft Excel $^{\bullet}$ for Windows ${ }^{\circledR} 10$.

O tratamento estatístico foi realizado por intermédio do programa Statistical Package for the Social Sciences - IBM SPSS ${ }^{\odot}$ 16.0. Após a determinação da normalidade da distribuição dos dados, por meio do teste de Kolmogorov-Smirnov, foi aplicado o teste de correlação de Pearson e os valores de $\mathrm{p}<0,05$ foram considerados significativos.

Este estudo passou pelo Comitê de Ética em Pesquisa com Seres Humanos da Universidade Região de Joinville - Univille, sob parecer número 4.392.423.

QUADRO 1 - Afirmativas que compõem o instrumento de pesquisa e as categorias de análise correspondentes.

\begin{tabular}{|c|c|c|}
\hline Categorias & Número & Questionário \\
\hline \multirow{4}{*}{ Aprendizado } & 1 & $\begin{array}{l}\text { A aula virtualizada é uma ferramenta negativa para a formação acadêmica } \\
\text { durante a pandemia do COVID-19. }\end{array}$ \\
\hline & 2 & $\begin{array}{l}\text { A aula virtualizada é uma ferramenta positiva para a formação acadêmica } \\
\text { durante a pandemia do COVID-19. }\end{array}$ \\
\hline & 3 & $\begin{array}{l}\text { As atividades remotas dificultaram minha participação nas aulas durante a } \\
\text { pandemia do COVID-19. }\end{array}$ \\
\hline & 4 & $\begin{array}{l}\text { As atividades remotas dificultaram o meu aprendizado e a assimilaçáo dos } \\
\text { conteúdos durante a pandemia do COVID-19. }\end{array}$ \\
\hline \multirow{4}{*}{ Caminho profissional } & 5 & $\begin{array}{l}\text { As atividades remotas me estimularam a adquirir novas habilidades/novos } \\
\text { hábitos, podendo ser um diferencial para a minha atuaçáo profissional futura. }\end{array}$ \\
\hline & 6 & $\begin{array}{l}\text { As atividades remotas prejudicaram a minha atuação profissional, pela falta das } \\
\text { aulas práticas. }\end{array}$ \\
\hline & 7 & $\begin{array}{l}\text { As atividades remotas prejudicaram a minha atuaçáo profissional, pela falta de } \\
\text { contato presencial com os professores. }\end{array}$ \\
\hline & 8 & $\begin{array}{l}\text { A experiência de aprendizagem durante a COVID-19 vai influenciar na minha } \\
\text { didática como profissional futuramente. }\end{array}$ \\
\hline \multirow{4}{*}{ Reconhecimento Intrínseco } & 9 & Estou abatido(a)/ desanimado(a) com a organização das aulas virtualizadas. \\
\hline & 10 & $\begin{array}{l}\text { Consegui me adaptar com as aulas virtualizadas e tornei oportuno para me } \\
\text { desenvolver como estudante, durante essa pandemia de COVID-19. }\end{array}$ \\
\hline & 11 & Estou saturado(a) com as aulas virtualizadas. \\
\hline & 12 & A pandemia me preparou para ser um profissonal resiliente. \\
\hline
\end{tabular}




\section{Resultados}

$\mathrm{Na}$ caracterização da amostra foi possível

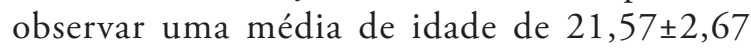
entre os respondentes. Contudo, outros dados também foram considerados importantes para distinguir o referido grupo, como a quantidade exata de participantes entre os gêneros, o perfil de idade e o período de formação acadêmica
(TABELA 1). Assim, verificou-se nos resultados apresentados na TABELA 1, uma associação (teste de Pearson) positiva moderada de 0,57 ( $\mathrm{p}$-valor $<0,003)$ entre a idade e o período de formação acadêmica, indicando uma prevalência do intervalo de idades correspondente aos mais jovens para os anos iniciais do curso.

TABELA 1 - Caracterização do grupo amostral.

\begin{tabular}{ccc}
\hline Gênero & $\mathbf{n}$ & $\%$ \\
\hline Feminino & 58 & 52,73 \\
Masculino & 52 & 47,27 \\
\hline Idade & & \\
\hline 18 a 21 & 66 & 60 \\
22 a 25 & 31 & 28,2 \\
26 a 29 & 13 & 11,8 \\
\hline Ano do curso & & \\
\hline $1^{\text {a série }}$ & 23 & 21 \\
2a série & 30 & 27,1 \\
3a série & 28 & 25,5 \\
4a série & 18 & 16,4 \\
5 série & 11 & 10 \\
\hline
\end{tabular}

Na TABELA 2 encontramos os dados referentes às questóes respondidas pelos participantes que foram divididas em três categorias, sendo que cada domínio representa um eixo relacionado à percepção da formação profissional de Educação Física durante as aulas remotas, devido às circunstâncias da pandemia do COVID-19.

De acordo com os resultados evidenciados na TABELA 2, podemos constatar que na categoria "Aprendizado", prevaleceram as respostas de "[2] concordo" para as questóes três $(40 / 36,5 \%)$ e quatro (45/41\%), de "[3] não concordo nem discordo" para a questão dois (37/33,6\%), e "[4] discordo" para a questão um $(38 / 34,5 \%)$. Em relação ao domínio "Caminho Profissional", verificou-se o predomínio do score "[2] concordo" para todas as questóes (5,6,7 e 8). Por fim, no componente "Reconhecimento Intrínseco", os resultados demonstraram maior frequência para as respostas de "[2] concordo" para as questóes nove $(34 / 31 \%)$ e doze $(48 / 43,6 \%)$, de "[4] discordo" para a questão dez (36/32,7\%), e de "[1] concordo plenamente" para a questão onze $(50 / 45,6 \%)$.

Com o objetivo de analisar o grau de associação linear entre as variáveis, a TABELA 3 apresenta os principais valores obtidos com o Teste de Coeficiente de Correlação de Pearson (r) em cada categoria.

Desta forma, é possível perceber na categoria "Aprendizado" a existência de uma correlação positiva moderada de 0,53 com p-valor $<0,002$ associando as variáveis 3 e 4.

Em relação ao domínio "Caminho Profissional", podemos identificar duas correlaçôes moderadas, sendo a primeira entre as variáveis 5 e 7 , resultando em uma associação negativa de $-0,41$ com $p$-valor $<0,0003$, e a segunda entre as variáveis 6 e 7, estabelecendo uma correlação positiva de 0,67 com p-valor $<0,003$.

No componente "Reconhecimento Intrínseco", mais duas associaçôes moderadas foram determinadas, sendo uma negativa de $-0,45$ com $\mathrm{p}$-valor $<0,005$ envolvendo as variáveis 9 e 10 , e outra positiva de 0,53 com p-valor $<0,002$ entre as variáveis 10 e 12. 
TABELA 2 - Estatística descritiva em categorias de ocorrência, utilizando a Escala Likert.

\begin{tabular}{|c|c|c|c|c|c|c|c|c|c|c|c|}
\hline \multicolumn{12}{|c|}{ Escala Likert } \\
\hline \multirow{3}{*}{ No Questóes } & \multicolumn{2}{|c|}{1} & \multicolumn{2}{|c|}{2} & \multicolumn{2}{|c|}{3} & \multicolumn{2}{|c|}{4} & \multicolumn{2}{|c|}{5} & \multirow{3}{*}{ Moda } \\
\hline & \multicolumn{2}{|c|}{$\begin{array}{l}\text { Concordo } \\
\text { plenamente }\end{array}$} & \multicolumn{2}{|c|}{ Concordo } & \multicolumn{2}{|c|}{$\begin{array}{l}\text { Náo concordo } \\
\text { nem discordo }\end{array}$} & \multicolumn{2}{|c|}{ Discordo } & \multicolumn{2}{|c|}{$\begin{array}{c}\text { Discordo } \\
\text { plenamente }\end{array}$} & \\
\hline & $\mathbf{n}$ & $\%$ & $\mathbf{n}$ & $\%$ & $\mathbf{n}$ & $\%$ & $\mathbf{n}$ & $\%$ & $\mathbf{n}$ & $\%$ & \\
\hline 1 & 6 & 5,5 & 22 & 20 & 37 & 33,6 & 38 & 34,5 & 7 & 6,4 & 4 \\
\hline 2 & 15 & 13,6 & 33 & 30 & 37 & 33,6 & 23 & 21 & 2 & 1,8 & 3 \\
\hline 3 & 26 & 23,5 & 40 & 36,5 & 16 & 14,5 & 22 & 20 & 6 & 5,5 & 2 \\
\hline 4 & 35 & 31,8 & 45 & 41 & 16 & 14,5 & 13 & 11,8 & 1 & 0,9 & 2 \\
\hline 5 & 12 & 10,9 & 35 & 31,9 & 29 & 26,4 & 27 & 24,4 & 7 & 6,4 & 2 \\
\hline 6 & 35 & 31,9 & 45 & 40,9 & 21 & 19,1 & 4 & 3,6 & 5 & 4,5 & 2 \\
\hline 7 & 31 & 28,1 & 50 & 45,5 & 20 & 18,2 & 8 & 7,3 & 1 & 0,9 & 2 \\
\hline 8 & 18 & 16,4 & 54 & 49,1 & 22 & 20 & 15 & 13,6 & 1 & 0,9 & 2 \\
\hline 9 & 24 & 21,8 & 34 & 31 & 32 & 29 & 17 & 15,5 & 3 & 2,7 & 2 \\
\hline 10 & 8 & 7,2 & 28 & 25,5 & 28 & 25,5 & 36 & 32,7 & 10 & 9,1 & 4 \\
\hline 11 & 50 & 45,6 & 38 & 34,5 & 16 & 14,5 & 5 & 4,5 & 1 & 0,9 & 1 \\
\hline 12 & 13 & 11,8 & 48 & 43,6 & 35 & 31,8 & 12 & 11 & 2 & 1,8 & 2 \\
\hline
\end{tabular}

TABELA 3 - Teste de correlação de Pearson ( $r$ ) em cada categoria.

\begin{tabular}{ccclc}
\hline Categorias & $\begin{array}{c}\text { Variáveis } \\
\text { (Questóes) }\end{array}$ & r & Correlaçáo & p-valor \\
\hline Aprendizado & 3 e 4 & 0,53 & Moderada & $0,002^{*}$ \\
Caminho profissional & 5 e 7 & $-0,41$ & Moderada & $0,0003^{*}$ \\
Caminho profissional & 6 e 7 & 0,67 & Moderada & $0,003^{*}$ \\
Reconhecimento intrínseco & 9 e 10 & $-0,45$ & Moderada & $0,005^{*}$ \\
Reconhecimento intrínseco & 10 e 12 & 0,53 & Moderada & $0,002^{*}$ \\
\hline
\end{tabular}

Diferenças significativas com p-valor $<0,005$.
Além disso, foi possível detectar semelhanças importantes nas variáveis, quando testadas entre categorias. Esses dados podem mostrar um pouco mais a respeito das informaçóes emitidas pelos participantes (TABELA 4).

Assim, quando analisadas as categorias "Aprendizado e Caminho Profissional", os resultados evidenciaram uma correlação positiva moderada de 0,42 com p-valor $<0,004$ entre as variáveis 1 e 7 , e uma correlação positiva moderada de 0,47 com p-valor<0,002 entre as variáveis 4 e 7 .

Entre os domínios "Aprendizado e Reconhecimento Intrínseco" ocorreu o maior número de correlaçóes, sendo a primeira positiva moderada de 0,44 com p-valor $<0,005$ para as variáveis 1 e 11 , a segunda positiva moderada de 0,41 com p-valor $<0,004$ entre as variáveis 4 e 9, e a terceira uma associação positiva moderada de 0,52 com p-valor $<0,001$ referente às variáveis 4 e 11 .

Nas categorias "Caminho Profissional e Reconhecimento Intrínseco", foi possível observar duas correlaçóes, sendo a primeira positiva moderada de 0,48 com p-valor $<0,003$ entre as variáveis 5 e 12, e por último uma correlaçáo positiva moderada de 0,48 com $\mathrm{p}$-valor $<0,003$ entre as variáveis 7 e 11. n: número amostral.

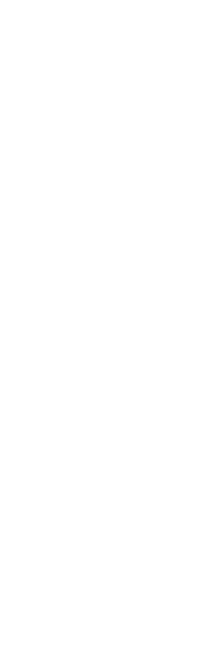


TABELA 4 - Teste de correlação de Pearson (r) entre as categorias.

Diferenças significativas com p-valor $<0,005$.

\begin{tabular}{ccccc}
\hline Categorias & $\begin{array}{c}\text { Variáveis } \\
\text { (Questóes) }\end{array}$ & r & Correlaçáo & p-valor \\
\hline Aprendizado e Caminho profissional & 1 e 7 & 0,42 & Moderada & $0,004^{*}$ \\
Aprendizado e Caminho profissional & 4 e 7 & 0,47 & Moderada & $0,002^{*}$ \\
Aprendizado e Reconhecimento Intrínseco & 1 e 11 & 0,44 & Moderada & $0,005^{*}$ \\
Aprendizado e Reconhecimento Intrínseco & 4 e 9 & 0,41 & Moderada & $0,004^{*}$ \\
Aprendizado e Reconhecimento Intrínseco & 4 e 11 & 0,52 & Moderada & $0,001^{*}$ \\
Caminho profissional e Reconhecimento Intrínseco & 5 e 12 & 0,48 & Moderada & $0,003^{*}$ \\
Caminho profissional e Reconhecimento Intrínseco & 7 e 11 & 0,48 & Moderada & $0,003^{*}$ \\
\hline
\end{tabular}

\section{Discussão}

De acordo com os dados obtidos, torna-se possível inferir que todas as categorias de análise propostas pelo instrumento de pesquisa deste estudo, foram impactadas com a mudança do ensino durante a pandemia do COVID-19. Este fato pode ser observado através dos resultados, justamente pela influência que cada domínio exerce sobre o outro, proporcionando um efeito cascata de forma contraproducente que pode ser constatado na estatística descritiva (Escala Likert) e pelo padrão de relacionamento entre as variáveis (Correlação de Pearson) desta população avaliada.

Desta forma, ao verificarmos o descontentamento dos acadêmicos de Educação Física em relação à participação nas aulas, assimilação de conteúdos e aprendizado ( $\mathrm{r} 0,53 \mathrm{com}$ p-valor $<0,002$ ), é possível perceber uma conexão com os achados referentes à percepçáo da formação profissional (r 0,67 com p-valor $<0,003$ ), no qual evidenciou principalmente a falta de interação advindas das aulas remotas. Além disso, é notável a repercussão dos fatos mencionados no componente subsequente, indicando uma força de relação moderada entre as variáveis correspondentes aos fatores intrínsecos $(\mathrm{r}-0,45 \mathrm{com}$ p-valor $<0,005)$.

Assim, corroborando com o desfecho da presente pesquisa, o estudo de Andreza et al. ${ }^{10}$, investigou a forma no qual o processo educacional está sendo afetado diante da atual situação do COVID-19 e os efeitos da aprendizagem por meio de atividades remotas. À vista disso, os estudantes revelaram-se insatisfeitos, apontando como negativo o seu rendimento. Compactuando dos mesmos argumentos do estudo mencionado, esse apontamento negativo pode estar relacionado a vários aspectos como a inacessibilidade parcial à internet, a falta de habilidades no uso de tecnologias e aos problemas pessoais e emocionais causados pelo isolamento social.

Este último aspecto, de acordo com Matias, Dominski e MARKs ${ }^{11}$, possibilita o agravamento de sentimentos que provavelmente produziráo resultados negativos em pessoas vulneráveis. Nesse sentido, buscar meios para controlar isso é fundamental, visto que chega a ser considerada uma necessidade durante o período de pandemia por amenizar o impacto psicológico do confinamento ${ }^{11}$.

Entretanto, torna-se importante destacar que para os alunos dos cursos de Educação Física (Licenciatura e Bacharelado) a experimentação prática é essencial na formaçáo profissional. A solidez desse argumento torna-se ainda maior, uma vez que perante a análise entre categorias, a variável 7 (relacionada a falta de contato presencial com os professores) obteve um grau de relação linear significativo em três possibilidades de associação com outras variáveis $(\mathrm{r}$ 0,42 com p-valor $<0,004$, r 0,47 com $p$-valor $<0,002$ e r 0,48 com p-valor $<0,003)$, indicando a relevância deste fator para o desempenho acadêmico.

Da mesma forma, no estudo de RamosMorcillo et al. ${ }^{12}$, que contou com a participaçáo de 32 alunos com idades entre 18 e 50 anos do curso de enfermagem, as aulas online tornaram-se um grande desafio. Os participantes preferiam a educação presencial, relatando que o método online possui limitaçóes, inviabilizando a compreensão 
com precisáo das atividades práticas.

Torna-se relevante ressaltar também, que as correlaçóes realizadas entre os eixos "Aprendizado e Reconhecimento Intrínseco" (r 0,44 com p-valor $<0,005$, r 0,41 com p-valor $<0,004$ e r 0,52 com $\mathrm{p}$-valor $<0,001$ ), apresentaram o maior número de associaçóes lineares significativas entre categorias, demonstrando uma variação conjunta da falta de entusiasmo dos alunos com as dificuldades encontradas no processo ensino-aprendizagem. Frente a esses dados, Rodrigues ${ }^{13}$, destaca que o uso de ferramentas virtuais no ensino trazem alguns pontos negativos como a falta de atenção, perda de foco e desinteresse.

Contudo, a pandemia expôs uma necessidade de transformação por meio de tecnologias remotas, a fim de manter a continuidade do planejamento letivo. Este cenário conduz alunos e professores para amplas reflexóes, no sentido de repensar estratégias para se adequar ao uso de novos modelos de ensino, reavaliar práticas tradicionais e descobrir novas habilidades sem afetar os princípios da educação5.

Partindo desta premissa, o presente estudo encontrou três associaçôes consideráveis $(\mathrm{r}-0,41$ com p-valor $<0,0003$, r 0,53 com p-valor $<0,002$ e r 0,48 com p-valor<0,003), com grau de relação linear moderado no que tange a adaptação com as aulas virtualizadas, a possibilidade de desenvolvimento como estudante e aquisição de novas habilidades a partir das atividades remotas, com a redução da percepção da necessidade do contato presencial e a capacidade de resiliência frente aos desafios impostos pela pandemia.

$\mathrm{O}$ ensino virtual é uma modalidade que rompe a relação habitual de sala de aula entre alunos e professores, fazendo com que o aluno seja mais independente e autônomo no seu processo de formaçáa ${ }^{14}$.

Este estudo possui algumas limitaçóes que devem ser mencionadas. Primeiramente, a representatividade e escolha da amostra, tendo em vista que os resultados obtidos retratam apenas a referida população. Ademais, a utilização de dados de forma auto-relatada, dificulta a precisão da informação coletada. Por esse motivo, torna-se inviável determinar relaçóes causais a partir de dados observacionais. Entretanto, os fatores que mitigam os eventuais vieses, estão pautados no rigor metodológico desta pesquisa.

Diante das evidências encontradas, foi possível constatar por intermédio do predomínio de associaçóes lineares significativas, que as atividades remotas dificultaram o processo ensinoaprendizagem para os acadêmicos dos cursos de Educação Física (Licenciatura e Bacharelado), da Universidade da Região de Joinville/SC - Univille, durante o período pandêmico. Neste contexto, torna-se importante analisar de que forma esta tecnologia/ferramenta está sendo inserida e efetivada institucionalmente, tendo em vista as características e necessidades de cada curso. Com base neste procedimento, a efetividade desta alternativa pedagógica poderá suprir de maneira mais adequada os anseios provindos do processo de formação acadêmica. 


\begin{abstract}
Physical Education in the Covid-19 era: remote classes in graduation during the pandemic.

This research aimed to analyze the impact of remote activities on the Physical Education courses at the University of the Region of Joinville/SC-Univille, during the pandemic period.The method adopted for the investigation was descriptive and transversal. A total of 110 students participated in the study, all of them from the Physical Education undergraduate courses at Univille. As a research instrument a questionnaire was used, organized in the Google Forms ${ }^{\circledR}$ platform, composed of 12 affirmative questions, in the Likert Scale model for measuring the answers. The questions were organized into three categories: Learning, Professional Path and Intrinsic Recognition. The data after being collected were organized for statistical treatment in the SPSS ${ }^{\circledast} 16.0$ program. After determining the normality of the data distribution, using the Kolmogorov-Smirnov test, Pearson's correlation test was applied and p-values $<0.05$ were considered significant. When performing the statistical analysis in each category, the results revealed five significant moderate linear associations. One in the "Learning" domain ( $p$-value $<0.002)$, two in the "Professional Path" ( $p$-value $<0.0003$ and p-value $<0.003$ ), and two in the "Intrinsic Recognition" ( $p$-value $<0.005$ and $\mathrm{p}$-value<0.002). For the analysis between categories, seven moderate correlations were found. Two in the "Learning and Professional Path"component (p-value $<0.004$ and p-value $<0.002)$, three in the "Learning and Intrinsic Recognition"(p-value $<0.005$, $p$-value $<0.004$ and p-value $<0.001$ ), and two in the "Professional Path and Intrinsic Recognition" with the same value ( $p$-value $<0.003$ ). Given the evidence found, it was possible to verify through the predominance of significant linear associations, that remote activities hindered the teaching-learning process for students of Physical Education courses, during the pandemic.
\end{abstract}

KeYwords: Pandemic; Remote Activities; Physical Education; Undergraduate.

\title{
Referências
}

1. Moreira LKR, Moreira LR, Soares MG. Educação Superior no Brasil: discussões e reflexóes. Educ Por Escr. 2018;9(1):134. Disponivel em: https://revistaseletronicas.pucrs.br/ojs/index.php/porescrito/article/view/29594.

2. Lopes LFM, Júnior RGN, Tunice LM da C. O Ensino Superior: caminhos, consensos e dissensos. ECCOM. 2020;11(22):399-406. Disponivel em: http://fatea.br/seer3/index.php/ECCOM/article/view/1198/1120.

3. Lau H, Khosrawipour V, Kocbach P, Mikolajczyk A, Ichii H, Schubert J, et al. Internationally lost COVID-19 cases. J Microbiol Immunol Infect. 2020;53(3):454-8. Disponivel em: https:/www.sciencedirect.com/science/article/pii/ S1684118220300736?via\%3Dihub.

4. Santos CC dos, Coutinho EF, Paillard GAL, Moreira LO. Um relato sobre os desafios das atividades remotas em um curso de graduação presencial diante das medidas de prevenção contra o SARS-CoV-2. Rev Novas Tecnol Educ. 2020;18(1). Disponivel em: https://seer.ufrgs.br/renote/article/view/106039.

5. Bezerra IMP. Estado da arte sobre o ensino de enfermagem e os desafios do uso de tecnologias remotas em época de pandemia do Corona Vírus. Rev Bras Crescimento Desenvolv Hum. 2020;30(1):141-7. Disponivel em: https://pesquisa. bvsalud.org/portal/resource/pt/biblio-1094937.

6. Teles G, Soares DMR, Lima L, Loureiro RC. Docência e tecnologias digitais na formação de professores: planejamento e execução de aulas por licenciandos. Brazilian J Technol. 2020;3(2):73-84. Disponivel em: https://www.brazilianjournals. com/index.php/BJT/article/view/9459.

7. Soares TLF de S, De Santana ÍS, Comper MLC. Ensino remoto na pandemia de COVID-19: liçóes aprendidas em um projeto de extensão universitário. Dialogia. 2020;(36):35-48. Disponivel em: https://periodicos.uninove.br/dialogia/ article/view/18396.

8. Regueiro EMG, Vasconcelos ECLM, Gonçalves AC, Figueiredo MML, Vasconcelos EE, Belluzzo SS, et al. Ensino mediado por tecnologias no curso de Fisioterapia do Centro Universitário Barão de Mauá durante o período de pandemia da COVID-19. Rev Interdiscip Saúde Educ. 2020;1(1):107-19. Disponivel em: https://periodicos.baraodemaua.br/index. $\mathrm{php/cse/article/view/36.}$ 
9. Freitas DA, Santos EMS, Lima LVS, Miranda LN, Vasconcelos EL, Nagliate PC. Saberes docentes sobre processo ensinoaprendizagem e sua importância para a formação professional em saúde. Interface Commun Heal Educ. 2016;20(57):43748. Disponivel em: https://www.scielo.br/scielo.php?pid=S1414-32832016000200437\&script=sci_abstract\&tlng=pt.

10. Andreza RS, Alves EJS, Martins LH, Silva RH, Silva SDA, Nogueira TL, et al. Os impactos da Covid-19 na educação por meio do ensino remoto. Rev Interfaces Saúde Humanas Tecnol. 2020;8(3):630-5. Disponivel em: https://interfaces. leaosampaio.edu.br/index.php/revista-interfaces/article/view/840.

11. Matias T, Dominski FH, Marks DF. Human needs in COVID-19 isolation. J Health Psychol. 2020;25(7):871-82. Disponivel em: https://journals.sagepub.com/doi/full/10.1177/1359105320925149.

12. Ramos-Morcillo AJ, Leal-Costa C, Moral-García JE, Ruzafa-Martínez M. Experiences of nursing students during the abrupt change from face-to-face to e-learning education during the first month of confinement due to COVID-19 in Spain. Int J Environ Res Public Health. 2020;17(15):1-15. Disponivel em: https://pesquisa.bvsalud.org/controlecancer/ resource/pt/mdl-32751660?src=similardocs.

13. Rodrigues JLO. A utilização das redes sociais virtuais no ambiente acadêmico. Cad Prof Adm UNIMEP. 2017;7(1):80-100. Disponivel em: http://www.cadtecmpa.com.br/ojs/index.php/httpwwwcadtecmpacombrojsindexphp/article/view/145.

14. Dos Santos CM, Bulgarelli PT, Frichembruder K, Colvara BC, Hugo FN. Avaliação da qualidade de aprendizagem no ambiente virtual (Moodle) em saúde bucal, na perspectiva dos discentes. Rev ABENO. 2018;18(1):116-23. Disponivel em: https://revabeno.emnuvens.com.br/revabeno/article/view/433/0.

\begin{tabular}{r|r} 
ENDEREço & \\
Eduarda Eugenia Dias de Jesus & \\
Universidade Região de Joinville - UNIVILLE & Submetido: 03/05/2021 \\
Rua Paulo Malschitzki, s/n- Zona Industrial Norte & Aceito: 29/06/2021 \\
89219-710 - Joinville - SC - Brasil & \\
E-mail: eduardaeugenia3@gmail.com & \\
&
\end{tabular}

